\title{
Distributed Space-Time/Frequency Coding Schemes for Single-Carrier Frequency Division Multiple Access Systems
}

\author{
A. Y. Al-nahari, F. E. Abd El-Samie, and M. I. Dessouky \\ Department of Electronics and Electrical Communications, Faculty of Electronic Engineering, \\ Menoufia University, Menouf 32952, Egypt \\ Correspondence should be addressed to A. Y. Al-nahari, nahari76@gmail.com
}

Received 21 December 2010; Accepted 9 February 2011

Academic Editor: A. Maaref

Copyright ( 92011 A. Y. Al-nahari et al. This is an open access article distributed under the Creative Commons Attribution License, which permits unrestricted use, distribution, and reproduction in any medium, provided the original work is properly cited.

The single carrier-frequency division multiple access (SC-FDMA) system is a new system that was adopted in the standardization of the upcoming 3GPP long-term evolution (LTE). Designing diversity-achieving schemes for the SC-FDMA system is a challenging task. The codes adopted should not affect the peak-to-average power ratio (PAPR) among other constraints. In this paper, we consider the design of cooperative diversity schemes for SC-FDMA systems in the uplink direction. Specifically, two relay-assisted distributed space-time/frequency codes are proposed. The proposed distributed space-frequency code (SFC) achieves full spatial diversity in the uplink fast-fading channels, where a diversity of order three can be achieved. The proposed code keeps a low PAPR, which is a good feature of the system. A minimum mean square error (MMSE) decoder is used at the receiver of the destination node. Moreover, we propose a bandwidth-efficient distributed space-time code (STC) for slow-fading relay channels. A decodeand-forward (DF) protocol is used at the relay node, and the possibility of erroneous decoding is taken into account. Simulation results demonstrate the performance improvement of the proposed schemes.

\section{Introduction}

Future broadband wireless networks should meet stringent requirements such as high data rate services over dispersive channels with high transmission reliability. Since the wireless resources such as the bandwidth and power are very scarce, they cannot cope with the increasing demand for higher data rates. Furthermore, wireless channels suffer from several impairments such as fading, shadowing, and multiuser interference that can degrade the system performance. In order to achieve such high bit rates and at the same time to meet the Quality of Service (QoS) requirements, orthogonal frequency division multiple access (OFDMA) is considered a mature technique to mitigate the problem of frequency selectivity and intersymbol interference (ISI) with other well-known advantages $[1,2]$. However, OFDMA, as a multicarrier technique, suffers from two main drawbacks. One is the high PAPR, which is a critical problem, especially in the uplink direction. It is required for the mobile terminal to be of low cost and high power efficiency, which is difficult to achieve with the above constraint. In addition,
OFDMA suffers from carrier frequency offset. An alternative to OFDMA is the SC-FDMA, which is a promising solution adopted as an uplink multiple access scheme in 3GPP-LTE [3-5]. Due to its single carrier nature, it has a low PAPR, while keeping most of the advantages of OFDMA such as assigning different numbers of subcarriers to different users and applying adaptive modulation and coding.

It is well known that the multiple-input multiple-output (MIMO) systems can significantly improve the capacity and reliability of the communication over fading channels using spatial multiplexing and/or space-time coding. Applying MIMO techniques for the SC-FDMA system was considered in $[6,7]$. The antennas should be well separated to have uncorrelated fading across different antennas. However, it is difficult to equip the small mobile unit with more than one antenna with uncorrelated fading. Therefore, transmit diversity can only be achieved through user cooperation leading to what is known as cooperative diversity $[8,9]$. The main idea is that the users in the network share their resources (e.g., the power and bandwidth) and transmit, cooperatively. The seminal work in [9] has proposed a variety 


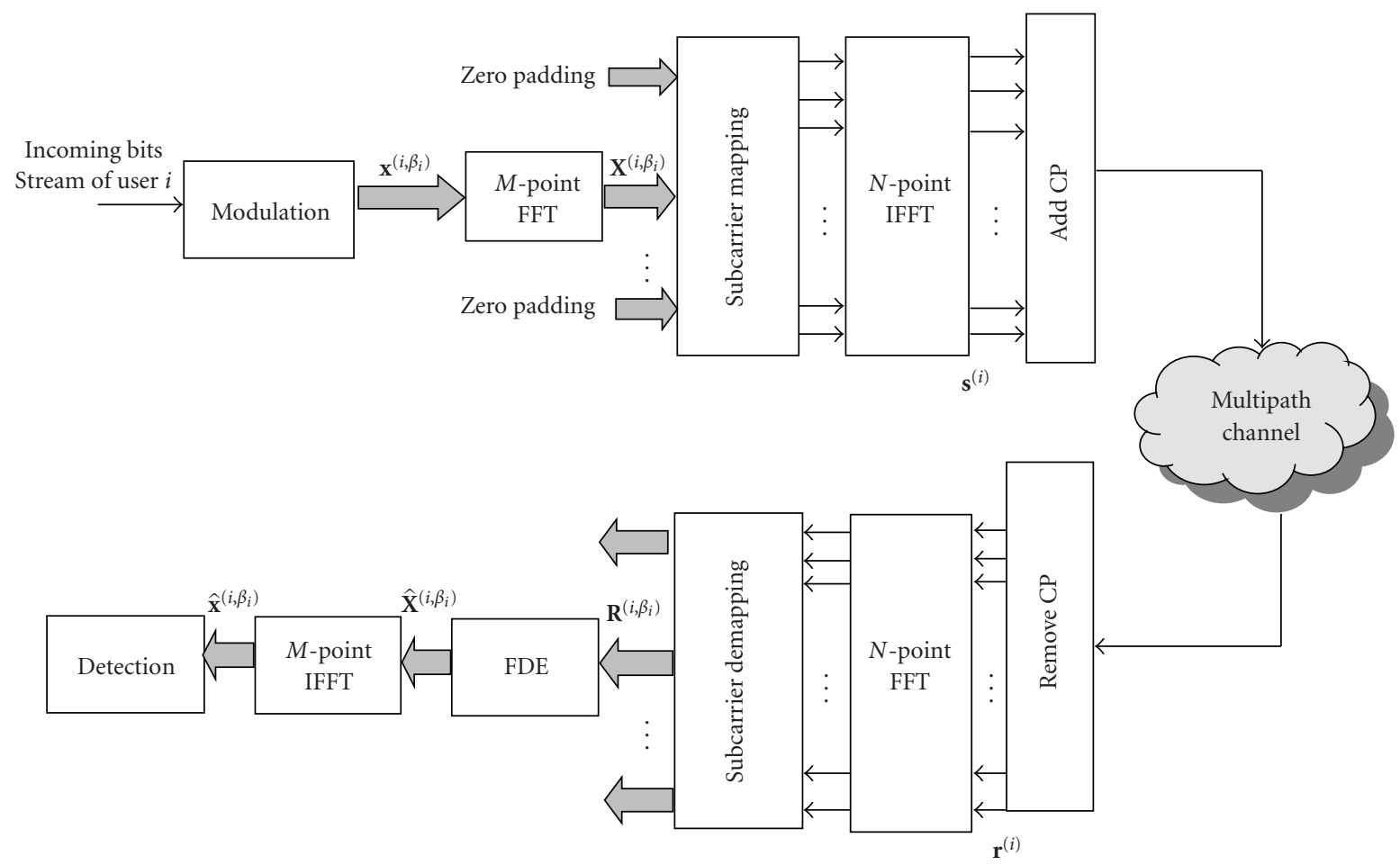

FIgURE 1: SC-FDMA system model of user $i$ for an SISO configuration.

of cooperative protocols using repetition coding. The most important among them are the amplify-and-forward (AF) protocol, and the DF protocol. In the AF protocol the relay simply receives its partner's information and retransmits it to the destination after amplification. In the DF protocol, the relay re-encodes the received signal in the first phase before retransmission to the destination in the second phase. In order to improve the spectral efficiency of the system, distributed space-time coding (DSTC) and distributed spacefrequency coding (DSFC) have been proposed [10-13]. Unfortunately, designing distributed space time/frequency codes for SC-FDMA systems is not straightforward. The codes should not affect the PAPR. In [14], the authors proposed a coded cooperation scheme to facilitate the time/frequency space diversity for the SC-FDMA system.

In this paper, we propose relay-assisted distributed spacetime/frequency codes for SC-FDMA systems in the uplink direction. The proposed distributed SFC is achieved in two phases, and the code does not affect significantly the PAPR of the transmitted signal. This code is suitable for fast-fading channels, where the channel gain changes from block to block. The coding is applied within each transmitted block. As the two coded symbols may experience different channel gains within one transmitted block, direct application of Alamouti decoding using maximum likelihood detection (MLD) over one symbol may degrade the performance especially with a large number of allocated subcarriers per user. Therefore, an MMSE decoder is used at the receiver to overcome this problem with a slight increase in the decoding complexity. Furthermore, we propose a distributed STC for the slow-fading channel environment. The proposed code is

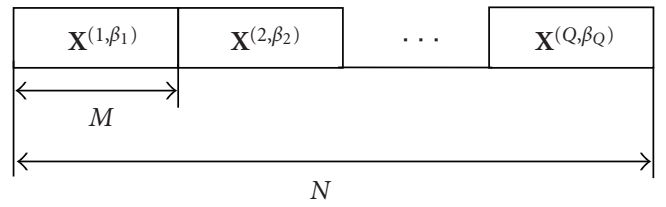

FIGURE 2: Illustration for the localized subcarriers allocation in the frequency domain for $Q$ users.

more spectrally efficient than the classical DSTC schemes. The proposed code achieves a transmission rate of $2 / 3$. For the two proposed codes, the DF protocol is considered and erroneous decoding at the relay node is taken into account. If the relay cannot decode correctly in the first phase, it remains idle in the second phase.

The rest of the paper is organized as follows. Section 2 presents the conventional SC-FDMA system model and the notations that are used throughout the paper. Section 3 presents the proposed distributed SFC with the DF protocol. The proposed distributed STC with the DF protocol is described in Section 4. The simulation results are provided in Section 5. Finally, Section 6 gives the concluding remarks.

\section{SC-FDMA System Model}

In this section, we present the system model for the conventional SC-FDMA system. In the following sections, the operations $[\cdot]^{\mathcal{T}},[\cdot]^{*}$, and $[\cdot]^{\mathscr{H}}$ stand for transpose, conjugate, and conjugate (Hermitian) transpose, respectively. $\mathbf{I}_{n}$ is an $n \times n$ identity matrix. The notation $\mathbf{x} \sim \mathcal{C} \mathcal{N}(\mu, \mathbf{C})$ denotes 
that random vector $\mathbf{x}$ is a circularly symmetric complex Gaussian random vector with mean $\mu$ and covariance matrix $\mathrm{C} ; \triangleq$ denotes equal by definition.

The SC-FDMA system model of user $i$ with a single-input single-output (SISO) configuration is shown in Figure 1. A group of $M$ modulated time-domain symbols that belong to user $i$, who will be given $M$ subcarriers after the fast Fourier transform (FFT) operation, can be represented as follows:

$$
\mathbf{x}^{\left(i, \beta_{i}\right)}=\left[x_{(i-1) M}^{\left(i, \beta_{i}\right)} x_{(i-1) M+1}^{\left(i, \beta_{i}\right)} \cdots x_{(i-1) M+M-1}^{\left(i, \beta_{i}\right)}\right]^{\mathcal{T}},
$$

where $x_{m}^{\left(i, \beta_{i}\right)}$ is the $m$ th time-domain symbol of user $i$, and $\beta_{i}$ denotes the frequency band that represents the subcarriers belonging to the $i$ th user. The block $\mathbf{x}^{\left(i, \beta_{i}\right)}$ is converted to the frequency domain. These $M$ frequency-domain symbols of user $i$ transmitted over the band $\beta_{i}$ are expressed as

$$
\mathbf{X}^{\left(i, \beta_{i}\right)}=\left[\begin{array}{lll}
X_{(i-1) M}^{\left(i, \beta_{i}\right)} & X_{(i-1) M+1}^{\left(i, \beta_{i}\right)} & \cdots X_{(i-1) M+M-1}^{\left(i, \beta_{i}\right)}
\end{array}\right]^{\mathcal{T}} .
$$

Localized subcarriers allocation is assumed in the following analysis as illustrated in Figure 2. A set of $M$ subcarriers is preallocated to each user, and the remaining $(N-M)$ subcarriers are zeroed, where $N$ denotes the total number of subcarriers in the system. Then, the signal is converted to the time domain using an inverse fast Fourier transform (IFFT) operation. The transmitted signal of user $i$ without cyclic prefix (CP) can be expressed as

$$
\mathbf{s}^{(i)}=\sqrt{P} \mathbf{F}_{N}^{\mathcal{H}} \mathbf{T}_{N, M} \mathbf{F}_{M} \mathbf{x}^{\left(i, \beta_{i}\right)},
$$

where $P$ is the total average transmitted power per symbol, $\mathbf{F}_{N}^{\mathcal{H}}$ is the $N$ points IFFT matrix, and $\mathbf{F}_{M}$ is the $M$ points FFT matrix. The $N \times M$ matrix $\mathbf{T}_{N, M}$ is the mapping matrix for subcarriers allocation. Each block is appended with a $\mathrm{CP}$ prior to transmission in order to prevent interblock interference (IBI) and to convert the linear convolution of the channel to a circular convolution. At the receiver, the $\mathrm{CP}$ is first removed, and the received signal in the time domain is given by

$$
\mathbf{r}^{(i)}=\sqrt{P} \mathbf{H} \mathbf{s}^{(i)}+\mathbf{n},
$$

where $\mathbf{H}$ is an $N \times N$ circulant matrix whose first column contains the channel impulse response appended by zeros. Therefore, $\mathbf{H}$ can be decomposed as follows:

$$
\mathbf{H}=\mathbf{F}_{N}^{\mathcal{H}} \boldsymbol{\Lambda} \mathbf{F}_{N},
$$

where $\Lambda$ is an $N \times N$ diagonal matrix containing the FFT of the channel impulse response. The signal goes through the FFT operation. Then, the received signal of the $i$ th user, after demapping, can be expressed as

$$
\mathbf{R}^{\left(i, \beta_{i}\right)}=\sqrt{P} \Lambda^{\left(\beta_{i}\right)} \mathbf{X}^{\left(i, \beta_{i}\right)}+\mathbf{N},
$$

where $\boldsymbol{\Lambda}^{\left(\beta_{i}\right)}=\operatorname{diag}\left\{H_{(i-1) M}, H_{(i-1) M+1}, \ldots, H_{(i-1) M+M-1}\right\}, H_{m}$ is the frequency response of the channel for the subcarrier $m$, and $\mathbf{N}$ is the additive noise vector that contains noise elements modeled as zero-mean complex Gaussian noise with variance $N_{0} / 2$ per dimension. Then, single tap equalization is performed in the frequency domain to retrieve the transmitted symbols. We will consider the MMSE equalizer. For MMSE equalization, the output signal is given by

$$
\begin{aligned}
\hat{\mathbf{X}}^{\left(i, \beta_{i}\right)} & =\mathbf{W}\left(\sqrt{P} \boldsymbol{\Lambda}^{\left(\beta_{i}\right)} \mathbf{X}^{\left(i, \beta_{i}\right)}+\mathbf{N}\right) \\
& =\sqrt{P} \mathbf{W} \boldsymbol{\Lambda}^{\left(\beta_{i}\right)} \mathbf{X}^{\left(i, \beta_{i}\right)}+\tilde{\mathbf{N}},
\end{aligned}
$$

where $\tilde{\mathbf{N}}=\mathbf{W N}$, and $\mathbf{W}$ is an $M \times M$ diagonal matrix whose $(k, k)$ element is given by

$$
\mathbf{W}(k, k)=\frac{\boldsymbol{\Lambda}^{\left(\beta_{i}\right)^{*}}(k, k)}{\left|\boldsymbol{\Lambda}^{\left(\beta_{i}\right)}(k, k)\right|^{2}+1 / \mathrm{SNR}},
$$

where SNR $=P / N_{0}$ is the average signal-to-noise ratio at the destination node. The decoding in SC-FDMA system is achieved in the time domain, so we need an $M$-point IFFT in order to demodulate the transmitted signal. The signal after the IFFT operation is given by

$$
\widehat{\mathbf{x}}^{\left(i, \beta_{i}\right)}=\mathbf{F}_{M}^{\mathcal{H}} \widehat{\mathbf{X}}^{(i, \beta)} .
$$

The recovered data $\widehat{\mathbf{x}}^{\left(i, \beta_{i}\right)}$ is sent to the decision operation at the final stage of SC-FDMA receiver.

\section{Cooperative Space-Frequency Coding for the SC-FDMA System}

In this section, we present the proposed cooperative diversity scheme for the SC-FDMA system based on DSFC. The proposed protocols utilize the SFC in the uplink SC-FDMA systems. We will consider Alamouti-like coding in which two users cooperate to send their information to the destination node. We will consider the DF protocol at the relay node.

3.1. Motivation and Cooperation Strategy. Consider the uplink transmission in the SC-FDMA system. The users in the system help each other to transmit, cooperatively, to the destination node in the second phase. Consider an example of two users sharing $N$ subcarriers that are divided equally between them. Specifically, user 1 is given $N_{1}$ subcarriers, which constitute a band $\beta_{1}$, and user 2 is given $N_{2}$ subcarriers, which constitute a band $\beta_{2}$, where $N=N_{1}+N_{2}$. The system has two phases as illustrated in Figure 3 . In the first phase, both users 1 and 2 send their own information on their corresponding subcarriers to the destination node (base station). Meanwhile, each user overhears his partner's information. The transmitted signals form user 1 and user 2 are denoted by the vectors $\mathbf{X}_{1}^{\left(1, \beta_{1}\right)}$ and $\mathbf{X}_{1}^{\left(2, \beta_{2}\right)}$, respectively. The transmitted signals in the frequency domain are also illustrated in Table 1, where $I_{i, j}$ is a random variable that represents the state of the $i$ th relay, whether it has decoded, correctly, at time slot $j$, or not.

In the second phase, user 1 sends his own data on the band $\beta_{1}$ and his partner's data (if he received correctly) on the band $\beta_{2}$. Similarly, in phase 2 , user 2 sends the processed information of his own data on the band $\beta_{1}$ and 


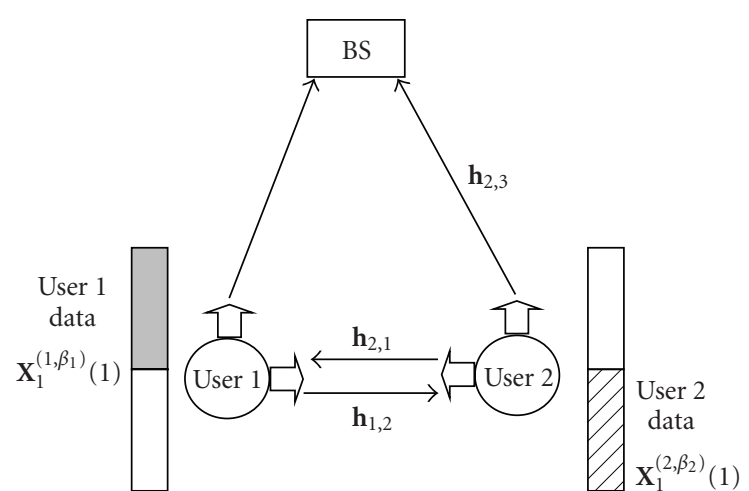

(a) Listening phase

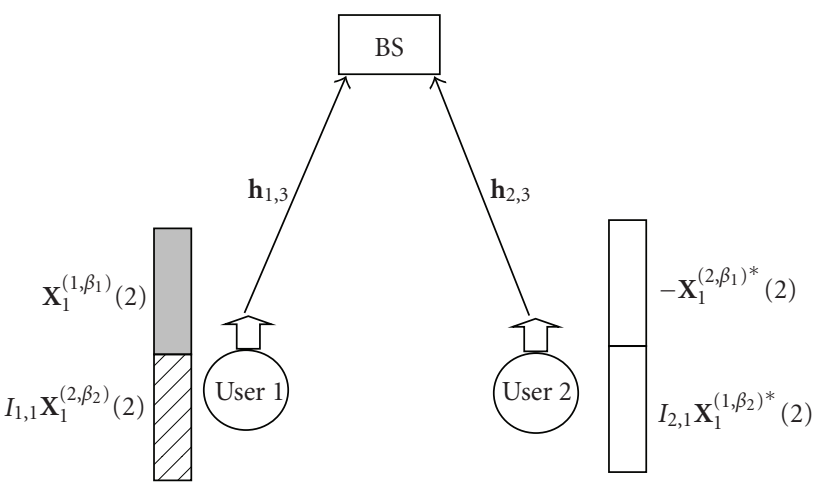

(b) Cooperation phase

FIgURe 3: Two cooperative users in the uplink SC-FDMA network. (a) Phase I, (b) Phase II.

his partner's processed data (if he received correctly) on the band $\beta_{2}$ as shown in Figure 3. This arrangement is made so as to constitute the modified version of the Alamouti coding scheme. In other words, the transmitted signals form the two users on both bands $\beta_{1}$ and $\beta_{2}$ in the second phase constitute Alamouti-like coding scheme in which two vectors are coded instead of two symbols. As will be shown later, the code construction does not affect the PAPR of the transmitted signal of user 1 . There is only a slight increase in the PAPR for the transmitted signals from user 2, but it is still less than that for other space-frequency coding schemes proposed in the literature.

As mentioned above, the signals transmitted in the second phase from both users constitute an Alamouti-like SFC, which achieves a diversity of order two. Furthermore, the signal transmitted in the first phase can also be exploited and combined with the signal received in the second phase at the receiver of the destination node. In the following analysis, we distinguish between two protocols regarding the received signal at the destination node in the first phase.

Protocol I. In the first phase, each user transmits his information to his partner. The destination node ignores the signals in this phase. In the second phase, in the case of correct decoding, both users communicate with the destination node to form the SFC on the bands $\beta_{1}$ and $\beta_{2}$. This is motivated by Protocol III proposed in [15]. As indicated in [15], the destination terminal may be engaged in data transmission to another terminal during the first time slot. Hence, the transmitted signal is received only at the relay terminal in the first phase.

Protocol II. This protocol differs from Protocol I in that the signals transmitted in the first time slot are received by the destination node and combined with the coded signals in the second time slot. In the case of a fast fading channel, the channel state changes from block to block. Therefore, a diversity of order three can be obtained as will be explained later. Further investigation of this type of nonorthogonal transmission to increase the spatial diversity was presented in [16].

3.2. Cooperative SFC for the SC-FDMA System with the DF Protocol. In this subsection, the proposed distributed SFC with the DF protocol is considered, where each terminal is equipped with a single antenna. Table 1 summarizes the transmitted signals during the two phases. In the following analysis, we will consider only one way cooperation cycle in which user 1 is the source node and user 2 acts as a relay node. The coding is applied only over the band $\beta_{1}$ allocated to user 1. Note that the same procedure explained in Section 3.1 and Table 1 to constitute the modified version of the Alamouti scheme is applied here, but using only the band $\beta_{1}$ for both users. The relay node performs decoding for the received vector in the first phase. The relay is assumed to be able to decide whether it has decoded correctly or not. This can be achieved through the use of error-detecting codes such as cyclic redundancy check (CRC) codes $[17,18]$.

In the first time slot $(t=1)$, the source node broadcasts its information to the destination and relay nodes. The received signals at the destination and the relay are given by

$$
\begin{aligned}
& \mathbf{R}_{B}^{\left(1, \beta_{1}\right)}(1)=\sqrt{P_{1}} \Lambda_{1,3}^{\left(\beta_{1}\right)} \mathbf{X}_{1}^{\left(1, \beta_{1}\right)}(1)+\mathbf{N}_{1,3}(1), \\
& \mathbf{R}_{U 2}^{\left(1, \beta_{1}\right)}(1)=\sqrt{P_{1}} \Lambda_{1,2}^{\left(\beta_{1}\right)} \mathbf{X}_{1}^{\left(1, \beta_{1}\right)}(1)+\mathbf{N}_{1,2}(1),
\end{aligned}
$$

where $P_{1}$ denotes the average transmitted power per symbol at the source node, $\mathbf{X}_{1}^{\left(1, \beta_{1}\right)}(1)$ is the frequency domain vector that belongs to user 1 transmitted in the first time slot on the band $\beta_{1}$, and $\Lambda_{1, j}^{\left(\beta_{1}\right)}, j=2,3$, are $M \times M$ diagonal matrices representing the frequency response of the channels between the source node and both the relay and destination nodes, $\mathbf{N}_{i, j}(1) \sim \mathcal{C} \mathcal{N}\left(\mathbf{0}, N_{0} \mathbf{I}_{M \times M}\right)$.

In the second time slot $(t=2)$, the source node transmits the first column $\left(\mathbf{X}_{1}^{\left(1, \beta_{1}\right)}\right)$ of the distributed SFC on the frequency band $\beta_{1}$. Meanwhile, if the relay has decoded correctly, it will forward the processed information to the destination on the frequency band $\beta_{1}$. Therefore, the received signal at the destination node during the second time slot is given by

$$
\begin{aligned}
\mathbf{R}_{B}^{\left(1, \beta_{1}\right)}(2)= & \sqrt{P_{1}} \Lambda_{1,3}^{\left(\beta_{1}\right)} \mathbf{X}_{1}^{\left(1, \beta_{1}\right)}(2) \\
& +\sqrt{P_{2}} I_{2,1} \Lambda_{2,3}^{\left(\beta_{1}\right)} \tilde{\mathbf{X}}_{1}^{\left(1, \beta_{1}\right)}(2)+\mathbf{N}(2),
\end{aligned}
$$



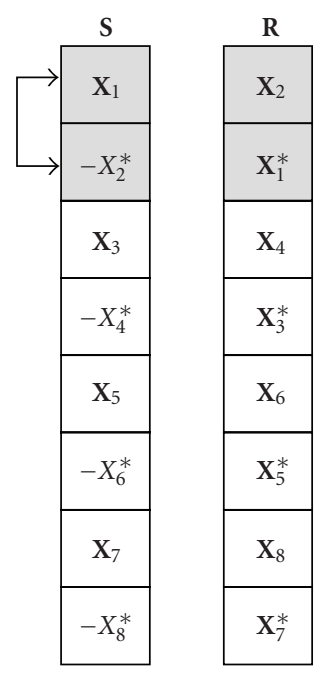

(a) Scheme I: Classical Scheme
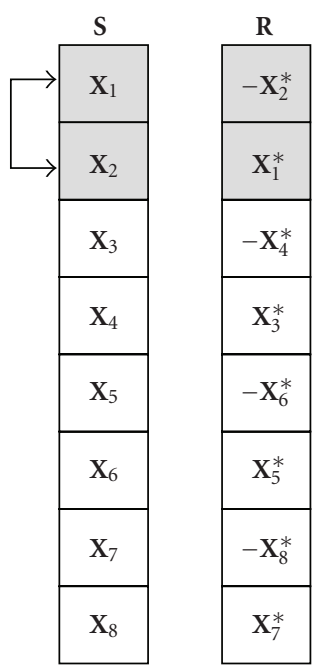

(b) Scheme II: Proposed in [7]
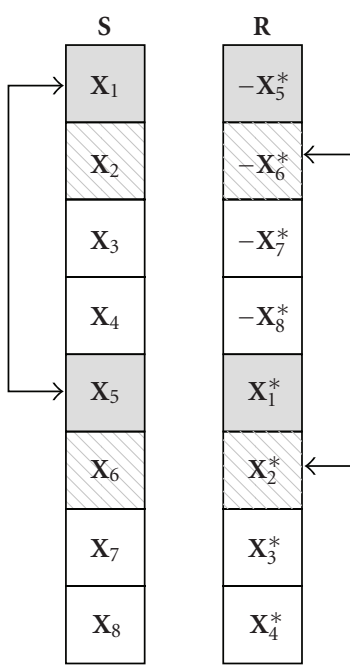

(c) Proposed coding scheme

FIGURE 4: Illustration of the proposed distributed SFC structure for user 1 for one-way cooperation on the band $\beta_{1}$ with $M=8$ compared to the classical SFC and the coding scheme presented in [7].

TABLE 1: The transmitted signals for the proposed distributed SFC using the DF protocol in the frequency domain.

\begin{tabular}{lcccc}
\hline Time slot $(t)$ & \multicolumn{1}{c}{ User 1 } & $\beta_{1}$ & User 2 \\
\hline 1 & $\beta_{1}^{\left(1, \beta_{1}\right)}(1)$ & - & - & $\beta_{2}$ \\
2 & $\mathrm{X}_{1}^{\left(1, \beta_{1}\right)}(2)$ & $I_{1,1} \mathbf{X}_{1}^{\left(2, \beta_{2}\right)}(2)$ & $-\mathbf{X}_{1}^{\left(2, \beta_{1}\right)^{*}}(2)$ & $\mathbf{X}_{1}^{\left(2, \beta_{2}\right)}(1)$ \\
\hline
\end{tabular}

where $P_{2}$ is the average transmitted power from the relay node, and $\tilde{\mathbf{X}}_{1}^{\left(1, \beta_{1}\right)}$ is the processed user 1's information received in the first phase. $I_{i, j}$ is a Bernoulli random variable that represents the state of the relay node $i$ (user 2 in this case) at time slot $j$. In other words, it indicates whether the relay has decoded correctly or not and is given by

$$
I_{i, j}=\left\{\begin{array}{ll}
1 & \text { correct decoding, } \\
0 & \text { error decoding, }
\end{array} \quad i, j=1,2 .\right.
$$

Such scheme guarantees the decoding at the destination node even if one of the channels was in deep fade. This is due to the orthogonality of the code matrix constructed at the receiver of the destination node. In other words, a maximum diversity gain of two is achieved even if the relay can not decode correctly in the first phase [10]. Let us partition the vector $\mathbf{X}_{1}^{\left(1, \beta_{1}\right)}$ (2) into two subvectors such that

$$
\mathbf{X}_{1}^{\left(1, \beta_{1}\right)}(2)=\left[\begin{array}{ll}
\mathbf{X}_{1,1}^{\left(1, \beta_{1}\right) \mathcal{T}} & \mathbf{X}_{1,2}^{\left(1, \beta_{1}\right) \mathcal{T}}
\end{array}\right]^{\mathcal{T}},
$$

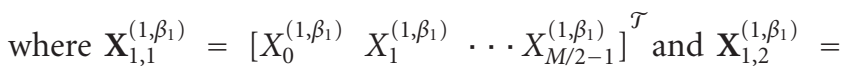

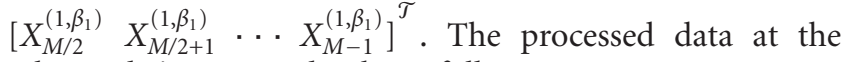
relay node is proposed to be as follows:

$$
\tilde{\mathbf{X}}_{1}^{\left(1, \beta_{1}\right)}(2)=\left[\begin{array}{ll}
-\mathbf{X}_{1,2}^{\left(1, \beta_{1}\right) \mathscr{H}} & \mathbf{X}_{1,1}^{\left(1, \beta_{1}\right) \mathscr{H}}
\end{array}\right]^{\mathcal{T}} .
$$

From (14) and (15), it can be seen that the transmitted codeword matrix from the source and relay nodes over the band $\beta_{1}$ in the second phase constitutes an Alamouti-like space-frequency code. Let us divide the received vector in (12) at the destination node into two sub-vectors as follows:

$$
\begin{aligned}
\mathbf{R}_{B, 1}^{\left(1, \beta_{1}\right)}(2)= & \sqrt{P_{1}} \Lambda_{1,3}^{\left(\beta_{1,1}\right)} \mathbf{X}_{1,1}^{\left(1, \beta_{1}\right)}(2) \\
& -\sqrt{P_{2}} I_{2,1} \Lambda_{2,3}^{\left(\beta_{1,1}\right)} \mathbf{X}_{1,2}^{\left(1, \beta_{1}\right)^{*}}(2)+\mathbf{N}_{1}(2), \\
\mathbf{R}_{B, 2}^{\left(1, \beta_{1}\right)}(2)= & \sqrt{P_{1}} \Lambda_{1,3}^{\left(\beta_{1,2}\right)} \mathbf{X}_{1,2}^{\left(1, \beta_{1}\right)}(2) \\
& +\sqrt{P_{2}} I_{2,1} \Lambda_{2,3}^{\left(\beta_{1,2}\right)} \mathbf{X}_{1,1}^{\left(1, \beta_{1}\right)^{*}}(2)+\mathbf{N}_{2}(2) .
\end{aligned}
$$

Note that the decoding of the received sub-vectors in (16) is similar to the steps presented in [19] if we assume approximately equal channel gains over the two coded subcarriers, that is, $\Lambda_{i, j}^{\left(\beta_{1,1}\right)} \approx \Lambda_{i, j}^{\left(\beta_{1,2}\right)}$ [20]. However, the channel response is no longer constant over the two subcarriers, especially if the two symbols encoded together are far separated. In the proposed code, the two symbols that are coded together are separated by $M / 2$ subcarriers as shown in Figure 4(c). In the classical SFC, the coding is performed over two adjacent subcarriers as shown in Figure 4(a). The scheme shown in Figure 4(b) was proposed in [7], and it was shown that this coding scheme has a lower PAPR compared to the classical scheme. The rationale behind the proposed 


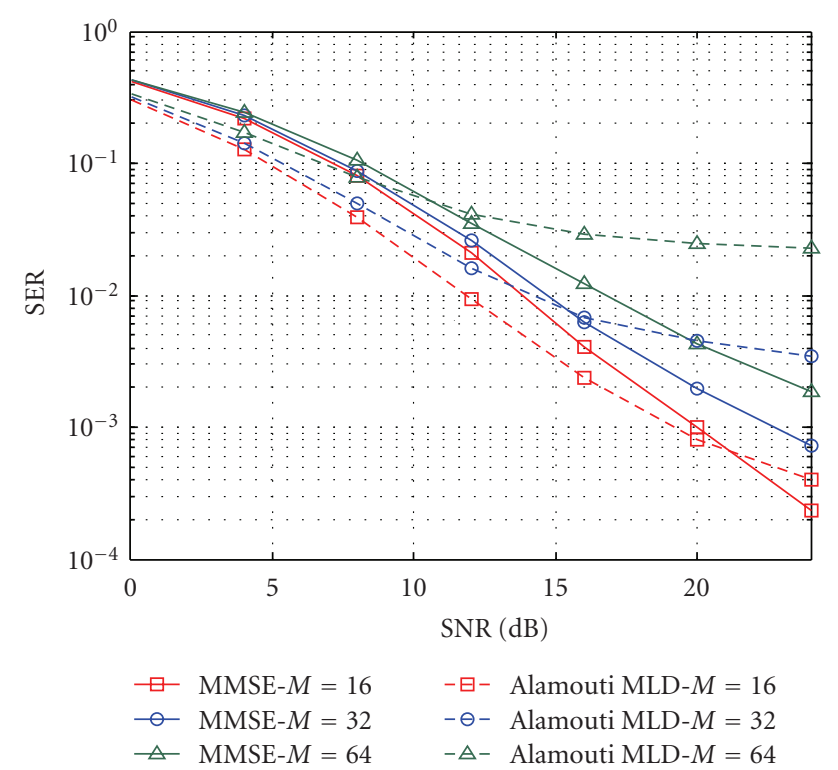

FIgURE 5: Performance comparison between MMSE decoding and Alamouti MLD decoding for the proposed distributed SFC with different number of subcarriers allocated to each user using Protocol I.

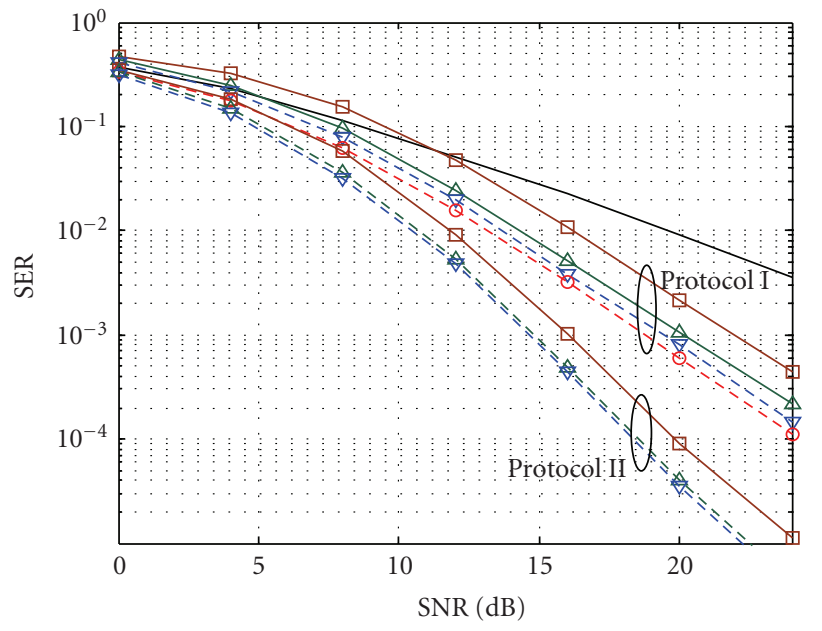

$$
\begin{aligned}
& - \text { SISO } \\
& \square-1 \mathrm{~dB} \text { interuser channel variance } \\
& \triangle 5 \mathrm{~dB} \text { interuser channel variance } \\
& -\nabla-10 \mathrm{~dB} \text { interuser channel variance } \\
& -\ominus-\text { MISO }
\end{aligned}
$$

FIGURE 6: SER versus SNR for the proposed distributed SFC for user 1 considering both Protocol I and Protocol II.

code structure, shown in Figure 4(c), is that the structure of the proposed code at the relay node does not break significantly the single carrier nature, and hence we get low PAPR, which is close to the uncoded SC-FDMA as will be shown in the simulation results. The code construction at the relay node can be considered as a circular shift of the original signal with simple conjugate and subtraction operations. So, it is expected that the PAPR of the proposed code is close to

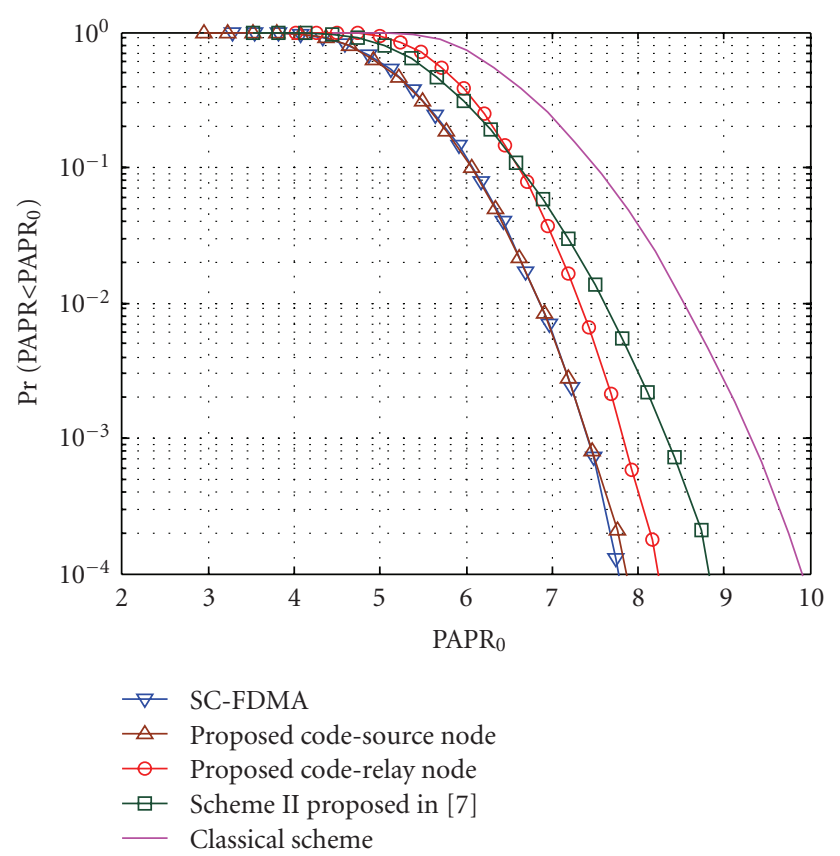

Figure 7: Performance comparison of the CCDF of the PAPR between the proposed distributed SFC, classical schemes, and uncoded SC-FDMA at the relay node.

the uncoded SC-FDMA. The performance of the proposed code gets worse with large $M$ when using Alamouti decoding with MLD for single symbol detection [19]. Therefore, we use a slightly more complex MMSE decoding at the receiver of the destination node. From (16), the received signal can be represented as

$$
\begin{aligned}
\underbrace{\left[\begin{array}{l}
\mathbf{R}_{B, 1}^{\left(1, \beta_{1}\right)}(2) \\
\mathbf{R}_{B, 2}^{\left(1, \beta_{1}\right)^{*}}(2)
\end{array}\right]}_{\mathbf{R}}= & \sqrt{\mathcal{P}} \underbrace{\left[\begin{array}{cc}
\boldsymbol{\Lambda}_{1,3}^{\left(\beta_{1,1}\right)} & -I_{2,1} \boldsymbol{\Lambda}_{2,3}^{\left(\beta_{1,1}\right)} \\
I_{2,1} \boldsymbol{\Lambda}_{2,3}^{\left(\beta_{1,2}\right)^{*}} & \boldsymbol{\Lambda}_{1,3}^{\left(\beta_{1,2}\right)^{*}}
\end{array}\right]}_{\mathbf{H}_{\mathrm{DF}}} \underbrace{\left[\begin{array}{c}
\mathbf{X}_{1,1}^{\left(1, \beta_{1}\right)} \\
\mathbf{X}_{1,2}^{\left(1, \beta_{1}\right)^{*}}
\end{array}\right]}_{\mathbf{X}} \\
& +\left[\begin{array}{l}
\mathbf{N}_{1}(2) \\
\mathbf{N}_{2}^{*}(2)
\end{array}\right],
\end{aligned}
$$

where we have defined $\mathcal{P} \triangleq P_{1}=P_{2}$. MMSE equalization can be used and the output of the equalizer can be written as

$$
\hat{\mathbf{X}}=\left(\mathbf{H}_{\mathrm{DF}}^{\mathcal{H}} \mathbf{H}_{\mathrm{DF}}+\frac{1}{\mathrm{SNR}}\right)^{-1} \mathbf{H}_{\mathrm{DF}}^{\mathcal{H}} \mathbf{R},
$$

where SNR $=P / N_{0}$ and $P=P_{1}+P_{2}$. Note that $\mathbf{H}_{\mathrm{DF}}$ is not an orthogonal matrix because $\boldsymbol{\Lambda}_{i, j}^{\left(\beta_{1,1}\right)} \neq \boldsymbol{\Lambda}_{i, j}^{\left(\beta_{1,2}\right)}$ for $i, j=1,2$. However, as far as the complexity is concerned, an inversion of matrices of order two, which contain the two coded symbols, is needed as seen in (17). The equalized signal $\hat{\mathbf{X}}$ is then transformed to the time domain for the final decoding.

It should be noted that (17) and (18) represent the received signals and the equalization process according to Protocol I described in Section 3.1. If we consider Protocol II, where the received signal in the first phase given in (10) is 
combined with the received signal in the second phase, which is given in (17), the combined signal is given as

$$
\begin{aligned}
\underbrace{\left[\begin{array}{c}
\mathbf{R}_{B, 1}^{\left(1, \beta_{1}\right)}(2)+\mathbf{R}_{B, 1}^{\left(1, \beta_{1}\right)}(1) \\
\mathbf{R}_{B, 2}^{\left(1, \beta_{1}\right)^{*}}(2)+\mathbf{R}_{B, 2}^{\left(1, \beta_{1}\right)^{*}}(1)
\end{array}\right]}_{\overline{\mathbf{R}}} \\
=\sqrt{\mathcal{P}} \underbrace{\left[\begin{array}{cc}
\boldsymbol{\Lambda}_{1,3}^{\left(\beta_{1,1}\right)}+\boldsymbol{\Lambda}_{1,3}^{\left(\beta_{1,1}\right)}(1) & -I_{2,1} \boldsymbol{\Lambda}_{2,3}^{\left(\beta_{1,1}\right)} \\
I_{2,1} \boldsymbol{\Lambda}_{2,3}^{\left(\beta_{1,2}\right)^{*}} & \boldsymbol{\Lambda}_{1,3}^{\left(\beta_{1,2}\right)^{*}}+\boldsymbol{\Lambda}_{1,3}^{\left(\beta_{1,2}\right)^{*}}(1)
\end{array}\right]} \\
\times \underbrace{\left[\begin{array}{c}
\mathbf{X}_{1,1}^{\left(1, \beta_{1}\right)} \\
\mathbf{X}_{1,2}^{\left(1, \beta_{1}\right)^{*}}
\end{array}\right]}_{\mathbf{X}}+\left[\begin{array}{c}
\tilde{\mathbf{N}}_{1} \\
\tilde{\mathbf{N}}_{2}
\end{array}\right] .
\end{aligned}
$$

We have used the time index for the channel frequency response in the first time slot to distinguish it from the received signals in the second time slot. $\widetilde{\mathbf{N}}_{1}$ and $\tilde{\mathbf{N}}_{2}$ are the new noise vectors. The MMSE criterion can be used and the equalized signal is given by

$$
\hat{\mathbf{X}}=\left(\overline{\mathbf{H}}_{\mathrm{DF}}^{\mathcal{H}} \overline{\mathbf{H}}_{\mathrm{DF}}+\frac{1}{\mathrm{SNR}}\right)^{-1} \overline{\mathbf{H}}_{\mathrm{DF}}^{\mathscr{H}} \overline{\mathbf{R}} .
$$

The PAPR for the user $i$ is defined as the ratio of the maximum power of the signal to the average power, and it is given by

$$
\operatorname{PAPR}^{(i)}=\frac{\max \left(\left|s_{n}\right|^{2}\right)}{(1 / N) \sum_{n=0}^{N-1}\left|s_{n}\right|^{2}}, \quad n=0,1, \ldots, N-1,
$$

where $s_{n}, n=0,1, \ldots, N-1$ are the elements of the transmitted time-domain vector given in (3).

\section{Cooperative STC for the SC-FDMA System}

In this section, we present the proposed bandwidth-efficient distributed STC that achieves better spectral efficiency than the classical scheme. The proposed scheme is illustrated in Table 2. The two-way cooperation cycle will last for three time slots. The channel is assumed to be constant over the three time slots.

In the first time slot, user 1 uses both his own subcarriers and his partner's subcarriers to transmit his information to the destination node. We argue that there is no practical limitation for the user to use both the subcarriers allocated to him and to his partner. At the same time $(t=1)$, user 2 is in the listening mode. At the second time slot $(t=2)$, user 2 uses both $\beta_{1}$ and $\beta_{2}$ to transmit his information to the destination node, while user 1 listens. At the third time slot, both users transmit on the two bands if they have decoded correctly in the previous time slots as shown in Table 2.

Due to the symmetry in cooperation in the two bands $\beta_{1}$ and $\beta_{2}$, we will consider only one cooperation cycle on one band (say $\beta_{1}$ ). Note that the transmitted signals on the

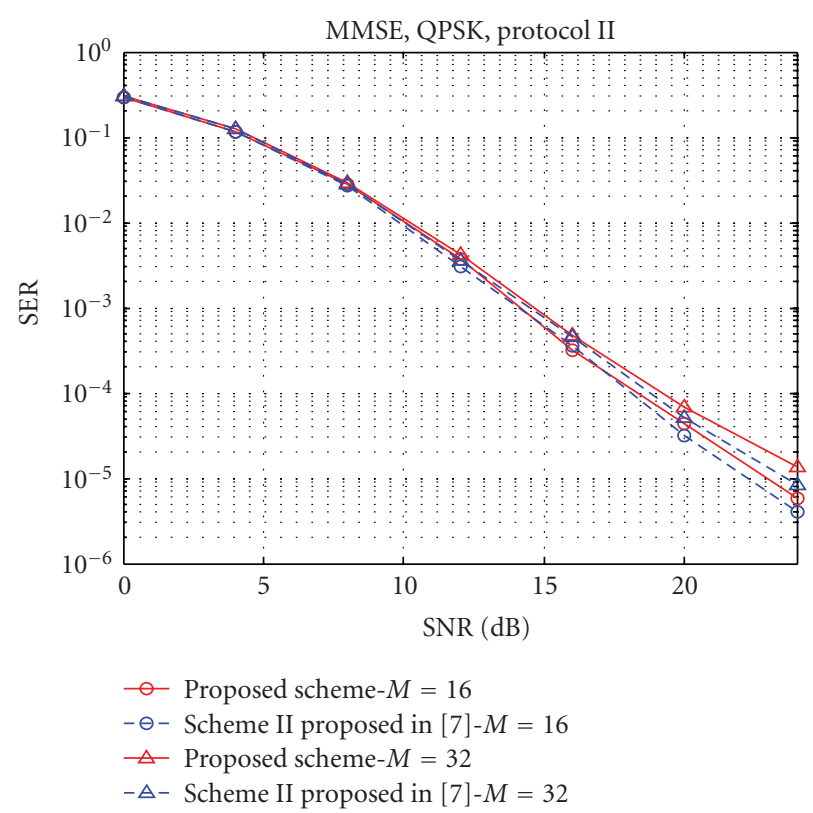

FIGURE 8: Performance comparison between the proposed scheme and the scheme proposed in [7] with a different number of subcarriers $M$.

band $\beta_{1}$ in Table 2 (columns 1 and 3 ) constitute an Alamoutilike STC. It is clear that every user sends two signal vectors in three time slots. Therefore, a transmission rate of $2 / 3$ is achieved. There are four possibilities in this scenario.

Case 1. Both users decode correctly, that is, $I_{2,1}=I_{1,2}=1$.

In this case, the received signals at the destination node can be expressed as

$$
\begin{aligned}
\mathbf{R}_{B}^{\left(\beta_{1}\right)}(1,2)= & \sqrt{P_{1}} \Lambda_{1,3}^{\left(\beta_{1}\right)} \mathbf{X}_{1}^{\left(1, \beta_{1}\right)}(1) \\
& +\sqrt{P_{2}} \Lambda_{2,3}^{\left(\beta_{1}\right)} \mathbf{X}_{1}^{\left(2, \beta_{1}\right)}(2)+\mathbf{N}(1)+\mathbf{N}(2), \\
\mathbf{R}_{B}^{\left(\beta_{1}\right)}(3)= & -\sqrt{P_{1}} \Lambda_{1,3}^{\left(\beta_{1}\right)} \mathbf{X}_{1}^{\left(2, \beta_{1}\right)^{*}}(3) \\
& +\sqrt{P_{2}} \Lambda_{2,3}^{\left(\beta_{1}\right)} \mathbf{X}_{1}^{\left(1, \beta_{1}\right)^{*}}(3)+\mathbf{N}(3),
\end{aligned}
$$

where $\mathbf{R}_{B}^{\left(\beta_{1}\right)}(1,2)$ is the linear combination of the received signals at the receiver of the destination node at time slots 1 and 2. It should be noted that the received signals in (22) and (23) contain the transmitted information of both users. The receiver performs Alamouti-like combining and maximum likelihood detection (MLD) as in [19]. The only difference here is the additional noise term in (22). We will investigate the effect of this variation compared to the classical scheme, where a single noise term is present in the simulation section.

Case 2. User 2 decodes correctly and user 1 does not, that is, $I_{2,1}=1, I_{1,2}=0$. In this case, user 1 does not send a signal in the third time slot in the band $\beta_{1}$. Therefore, the received 


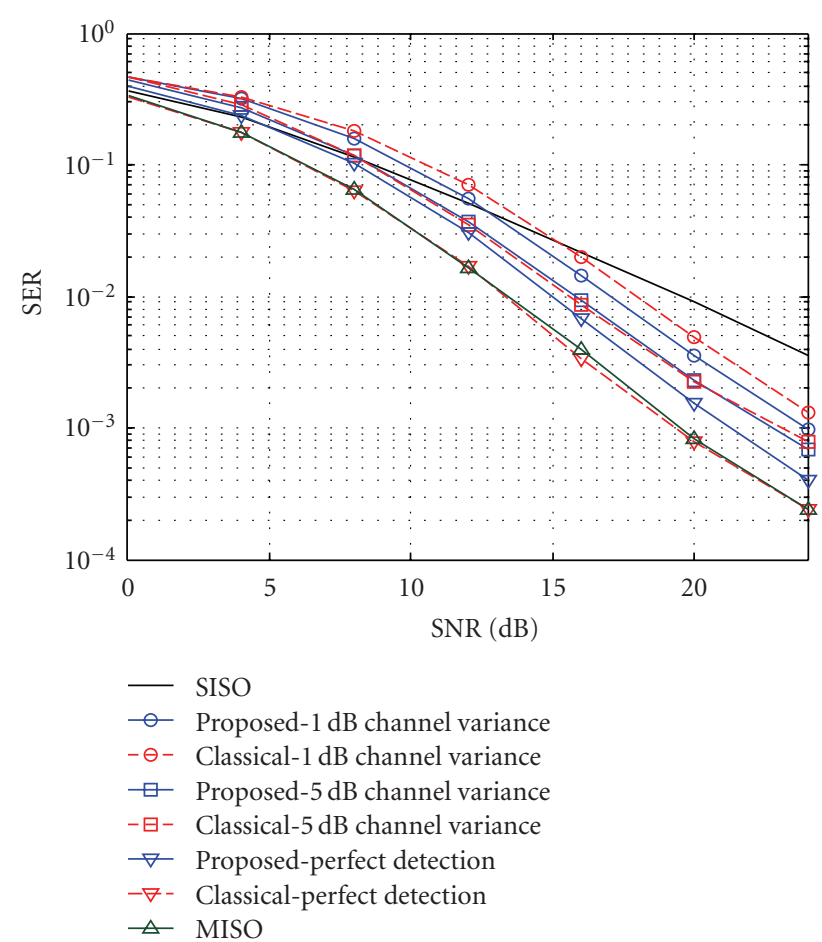

FIGURE 9: SER performance comparison between the proposed distributed STC and the classical scheme with different inter-user channel variances.

signals at the destination node are given by

$$
\begin{aligned}
& \mathbf{R}_{B}^{\left(\beta_{1}\right)}(1)=\sqrt{P_{1}} \Lambda_{1,3}^{\left(\beta_{1}\right)} \mathbf{X}_{1}^{\left(1, \beta_{1}\right)}(1)+\mathbf{N}(1), \\
& \mathbf{R}_{B}^{\left(\beta_{1}\right)}(2)=\sqrt{P_{2}} \Lambda_{2,3}^{\left(\beta_{1}\right)} \mathbf{X}_{1}^{\left(2, \beta_{1}\right)}(2)+\mathbf{N}(2), \\
& \mathbf{R}_{B}^{\left(\beta_{1}\right)}(3)=\sqrt{P_{2}} \Lambda_{2,3}^{\left(\beta_{1}\right)} \mathbf{X}_{1}^{\left(1, \beta_{1}\right)^{*}}(3)+\mathbf{N}(3),
\end{aligned}
$$

The receiver combines the received signals in (24) and (26) using MRC and makes decision for the signal vector $\mathbf{X}_{1}^{\left(1, \beta_{1}\right)}$ of user 1. Equation (25) is decoded separately.

Case 3. User 2 fails to decode whereas user 1 decodes correctly, that is, $I_{2,1}=0, I_{1,2}=1$. In this case, the received signals at the destination node during the first and second time slots are similar to that in (24) and (25). The received signal at the third time slot is given by

$$
\mathbf{R}_{B}^{\left(\beta_{1}\right)}(3)=-\sqrt{P_{1}} \Lambda_{1,3}^{\left(\beta_{1}\right)} \mathbf{X}_{1}^{\left(2, \beta_{1}\right)^{*}}(3)+\mathbf{N}(3) .
$$

Similar to Case 2, the receiver combines the signals in (25) and (27) using the maximal ratio combining (MRC) and makes decision for the vector $\mathbf{X}_{1}^{\left(2, \beta_{1}\right)}$ that belongs to user 2 . Equation (24) is decoded, separately.

Case 4. Both user 1 and user 2 fail to decode correctly, that is, $I_{2,1}=I_{1,2}=0$. In this case, the received signals at the destination node in the first and second time slots are similar to (24) and (25). The two users send nothing in the third time slot. The receiver decodes these two received signals, separately, and the decision variables in the frequency domain for users 1 and 2 are given by

$$
\begin{aligned}
& \hat{\mathbf{X}}_{1}^{\left(1, \beta_{1}\right)}=\boldsymbol{\Lambda}_{\mathbf{1}, 3}^{\left(\beta_{1}\right)^{*}} \mathbf{R}_{B}^{\left(1, \beta_{1}\right)}(1), \\
& \hat{\mathbf{X}}_{1}^{\left(2, \beta_{1}\right)}=\boldsymbol{\Lambda}_{2,3}^{\left(\beta_{1}\right)^{*}} \mathbf{R}_{B}^{\left(1, \beta_{1}\right)}(2) .
\end{aligned}
$$

In the above analysis, we have assumed symmetric interuser $\mathrm{SNR}$, that is, $\mathrm{SNR}_{1,2}=\mathrm{SNR}_{2,1}=\mathcal{P} / \mathrm{N}_{0}$. As will be shown in the simulation section, the proposed scheme achieves a diversity of order two, and the performance highly depends on the interuser channel quality.

It should be noted that for the receiver of the destination node to combine the received signals, the state of each node, whether it has decoded correctly or not, should be forwarded to the destination node. This can be accomplished by sending one bit from each user to the destination to indicate whether the user could decode the received signal from his partner, correctly, or not.

\section{Simulation Results}

In this section, we verify the performance of the proposed cooperative diversity schemes via computer simulations. The uplink direction of the SC-FDMA system is considered. A total of $N=512$ subcarriers is used in the simulation. The signal constellation is a quadrature phase shift keying (QPSK) constellation. Channel modeling is based on an exponentially decaying power delay profile. The operating bandwidth is $20 \mathrm{MHz}$, and the maximum excess delay of the channel $\left(\tau_{\max }\right)$ is chosen to produce a channel with 10 paths. The power of the channel paths is normalized to one. The CP is assumed to eliminate the ISI. It also converts the linear convolution of the channel impulse response to a circular convolution. The power is divided equally between the source node and the relay node. The SNR used in the simulation is defined as SNR $=\left(P_{1}+P_{2}\right) / N_{0}$, where $P_{1}=P_{2}$.

Figure 5 shows the symbol error rate (SER) performance of the proposed SFC using two decoding schemes: Alamouti MLD and the MMSE schemes. As shown from the figure, the performance of the MLD scheme starts to degrade at high SNR values due to the error floor, because the two symbols coded together experience different channel responses. As the number of the allocated subcarriers $M$ increases, the SER performance gets worse. Therefore, in the following simulation experiments, we will use the MMSE criterion.

Figure 6 shows the SER performance of the proposed distributed SFC with the DF protocol. For the case of the relay located at the midpoint between the source and the destination, all channels $(S \rightarrow D, S \rightarrow R, R \rightarrow D)$ are assumed to be of unit variance. For the case the relay is close to the source, the source-relay channel variance $\sigma_{s, r}^{2}$ is increased in the simulation. Figure 6 shows that as the relay node becomes closer to the source node, the performance is greatly improved, and the performance of the proposed scheme approaches the performance of the multiple-input singleoutput (MISO) system with two antennas at the transmitter side. Note also that the DF signaling at the relay is very 
TABLE 2: The transmitted signals in the frequency domain for the proposed distributed STC with the DF protocol.

\begin{tabular}{lcccc}
\hline Time slot $(t)$ & & User 1 & & User 2 \\
\hline 1 & $\beta_{1}$ & $\beta_{2}$ & $\beta_{1}$ & $\beta_{2}$ \\
2 & $\mathbf{X}_{1}^{\left(1, \beta_{1}\right)}(1)$ & $\mathbf{X}_{2}^{\left(1, \beta_{2}\right)}(1)$ & - & - \\
3 & - & - & $\mathbf{X}_{1}^{\left(2, \beta_{1}\right)}(2)$ & $\mathbf{X}_{2}^{\left(2, \beta_{2}\right)}(2)$ \\
& $-I_{1,2} \hat{\mathbf{X}}_{1}^{\left(2, \beta_{1}\right)^{*}}(3)$ & $-I_{1,2} \hat{\mathbf{X}}_{2}^{\left(2, \beta_{2}\right)^{*}}(3)$ & $I_{2,1} \hat{\mathbf{X}}_{1}^{\left(1, \beta_{1}\right)^{*}}(3)$ & $I_{2,1} \hat{\mathbf{X}}_{2}^{\left(1, \beta_{2}\right)^{*}}(3)$ \\
\hline
\end{tabular}

sensitive to the channel condition of the source-relay link. As can be seen from the figure, a diversity of order three can be achieved using Protocol II.

The complementary cumulative distribution functions (CCDFs) of the PAPR of the transmitted signals at the relay node for the proposed scheme, the classical scheme, and scheme II proposed in [7] are shown in Figure 7. It is clear from the figure that the proposed scheme achieves about $1.5 \mathrm{~dB}$ gain in PAPR compared to the classical scheme at a CCDF value of $10^{-3}$. Moreover, the proposed scheme achieves about $0.6 \mathrm{~dB}$ gain compared to scheme II proposed in [7]. Note that the transmitted signal of the proposed scheme at the source node achieves the same PAPR as in the case of uncoded SC-FDMA. Figure 8 compares the SER performance of the proposed SFC with the classical SFC (scheme II). As can be seen from the figure, the performance of the two schemes is the same at low SNR values. At high SNR values, there is about $0.2 \mathrm{~dB}$ loss for the proposed scheme compared to scheme II.

The SER performance comparison between the proposed distributed STC and the classical coding scheme is shown in Figure 9. The cooperative protocol of the classical scheme needs four time slots to transmit two blocks. Therefore, the transmission rate is $1 / 2$. Note that the proposed scheme offers a better performance than the direct transmission. The performance gain is further improved with the higher interuser channel variance. It can be seen that the slope of the curve of the proposed scheme is the same as that of MISO case, and thereby it achieves a diversity of order two.

It can also be seen that the proposed scheme has a better performance than the classical distributed STC, when all channel variances are equal to one. As the inter-user channel variance increases and other channels variances are equal to one, the performance loss of the proposed scheme increases compared to the classical scheme, because case 1 will be the dominant case, and the additional noise component in (22) makes this performance loss.

\section{Conclusions}

In this paper, we have proposed cooperative diversity schemes that are suitable for SC-FDMA systems in the uplink direction. The proposed distributed SFC achieves a full spatial diversity and keeps the low PAPR of the SCFDMA systems. The SER performance loss of the proposed code is approximately negligible compared to the classical scheme. As the classical simple Alamouti decoding degrades the performance, we have used the MMSE decoding with a small increase in the complexity. Furthermore, we have proposed a distributed STC that is suitable for slow fading channels. The proposed code achieves a transmission rate of $2 / 3$. Simulation results indicate that a better performance is achieved with small inter-user channel variances compared to the classical scheme. With a strong source-relay channel, the proposed code achieves a full diversity with a small performance loss.

\section{References}

[1] H. Sari and G. Karam, "Orthogonal division multiple access and its application to CATV networks," European Transactions on Telecommunications, vol. 9, no. 6, pp. 507-516, 1998.

[2] R. Laroia, S. Uppala, and J. Li, "Designing a mobile broadband wireless access network," IEEE Signal Processing Magazine, vol. 25, pp. 20-28, 2004.

[3] R. Dinis and D. Falconer, "A multiple access scheme for the uplink of broadband wireless systems," in Proceedings of the IEEE Global Telecommunications Conference (GLOBECOM '04), pp. 3808-3812, December 2004.

[4] H. G. Myung, J. Lim, and D. J. Goodman, "Single carrier FDMA for uplink wireless transmission," IEEE Vehicular Technology Magazine, vol. 1, no. 3, pp. 30-38, 2006.

[5] 3GPP TS 36.201 V 8.1.0, "LTE- Physical Layer-General description (Release 8)," November 2007.

[6] M. Noune and A. Nix, "Frequency-domain transmit processing for MIMO SC-FDMA in wideband propagation Channels," in Proceedings of the Wireless Communications and Networking Conference (WCNC '09), pp. 1-6, April 2009.

[7] D. Li, P. Wei, and X. Zhu, "Novel space-time coding and mapping scheme in single-carrier FDMA systems," in Proceedings of the IEEE International Symposium on Personal, Indoor and Mobile Radio Communications (PIMRC '07), pp. 1-4, September 2007.

[8] A. Sendonaris, E. Erkip, and B. Aazhang, "User cooperation diversity - part II: implementation aspects and performance analysis," IEEE Transactions on Communications, vol. 51, no. 11, pp. 1939-1948, 2003.

[9] J. Laneman, D. Tse, and G. W. Wornell, "Cooperative diversity in wireless networks: efficient protocols and outage behavior," IEEE Transactions on Information Theory, vol. 50, no. 12, pp. 3062-3080, 2004.

[10] J. N. Laneman and G. W. Wornell, "Distributed space-timecoded protocols for exploiting cooperative diversity in wireless networks," IEEE Transactions on Information Theory, vol. 49, no. 10, pp. 2415-2425, 2003.

[11] A. Y. Al-nahari, M. I. Dessouky, and F. E. Abd El-Samie, "Cooperative space-time coding with amplify-and-forward relaying," Journal of Signal Processing Systems.

[12] K. G. Seddik and K. J. R. Liu, "Distributed space-frequency coding over broadband relay channels," IEEE Transactions on Wireless Communications, vol. 7, no. 11, pp. 4748-4759, 2008. 
[13] A. Y. Al-Nahari, K. G. Seddik, F. E. Abd El-Samie, and M. I. Dessouky, "Cooperative space-frequency coding for broadband relay channels," in Proceedings of the International Conference on Computer Engineering and Systems (ICCES '09), Cairo, Egypt, December 2009.

[14] J. Niu and I. T. Lu, "Coded cooperation on block-fading channels in single carrier FDMA systems," in Proceedings of the IEEE Global Communications Conference(GLOBECOM '07), pp. 4339-4343, November 2007.

[15] R. U. Nabar, H. Bölcskei, and F. W. Kneubühler, "Fading relay channels: performance limits and space-time signal design," IEEE Journal on Selected Areas in Communications, vol. 22, no. 6, pp. 1099-1109, 2004.

[16] I. Krikidis, J. Thompson, and S. Mclaughlin, "On the diversity order of non-orthogonal amplify-and-forward over blockfading channels," IEEE Transactions on Wireless Communications, vol. 9, no. 6, pp. 1890-1900, 2010.

[17] P. Merkey and E. C. Posner, "Optimal cyclic redundancy codes for noise channels," IEEE Transactions on Information Theory, vol. 30, no. 3, pp. 865-867, 1984.

[18] A. K. Sadek, W. Su, and K. J. R. Liu, "Multinode cooperative communications in wireless networks," IEEE Transactions on Signal Processing, vol. 55, pp. 341-355, 2007.

[19] S. M. Alamouti, "A simple transmit diversity technique for wireless communications," IEEE Journal on Selected Areas in Communications, vol. 16, no. 8, 1998.

[20] K. F. Lee and D. B. Williams, "Space-frequency transmitter diversity technique for OFDM systems," in Proceedings of the IEEE Global Telecommunication Conference (GLOBECOM '00), vol. 3, pp. 1473-1477, November 2000. 

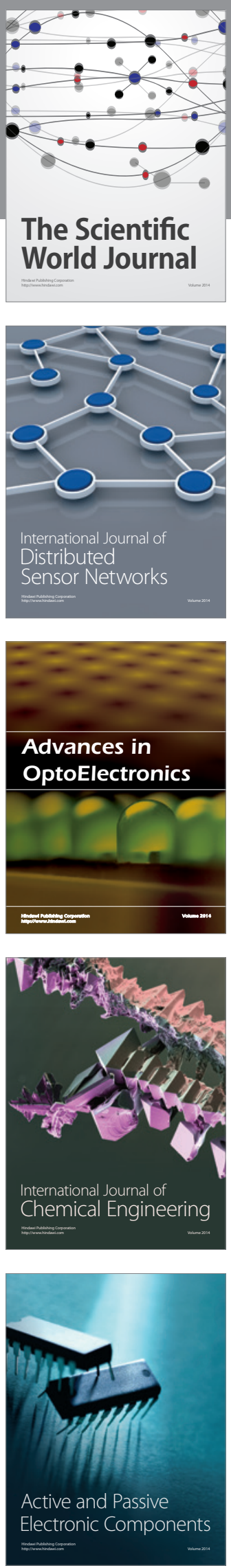
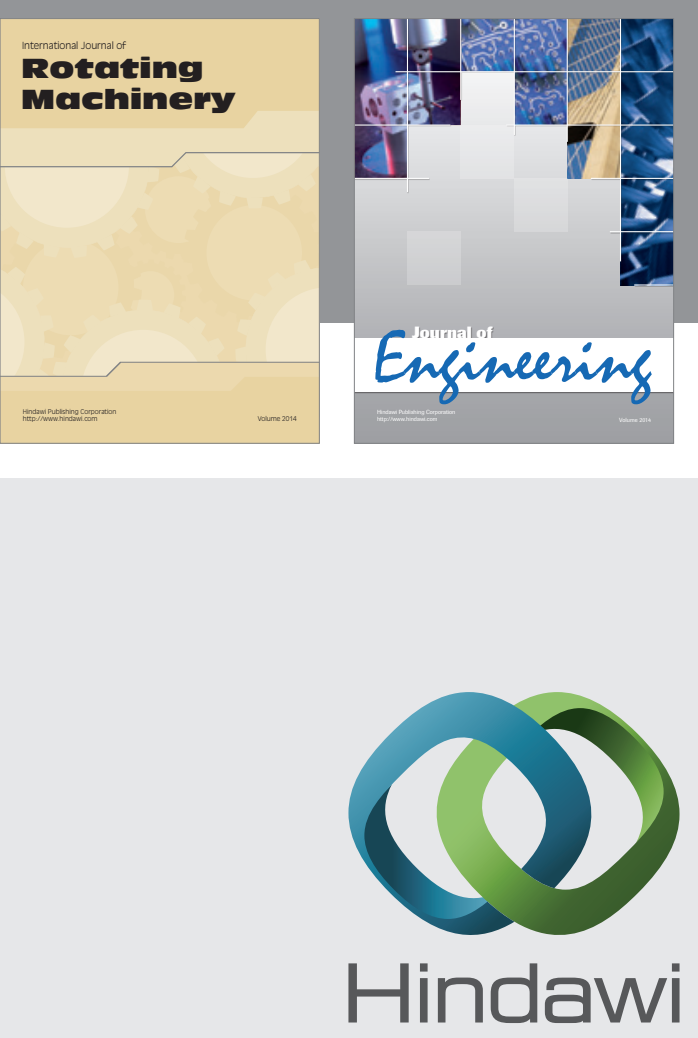

Submit your manuscripts at

http://www.hindawi.com
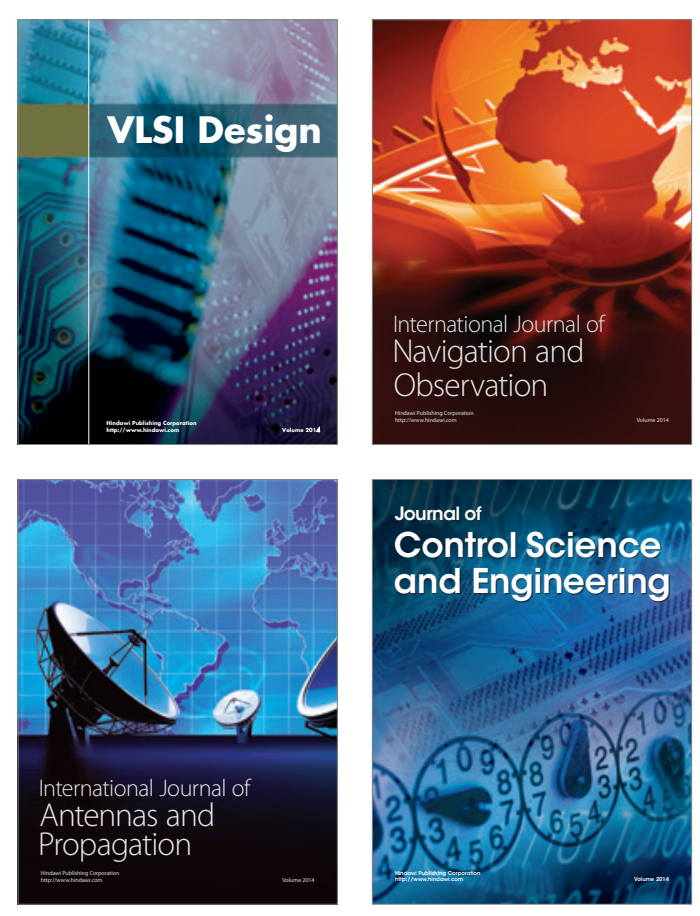
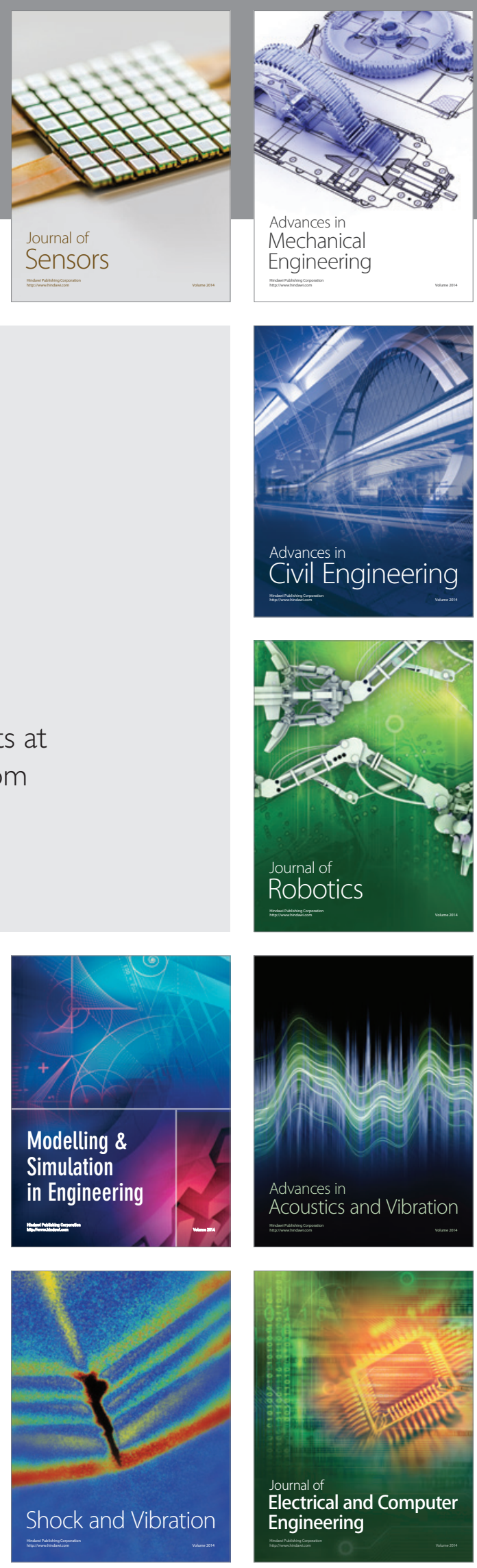